\title{
ARTICULACIÓN ENTRE EL NIVEL MEDIO Y LA UNIVERSIDAD: UNA PROPUESTA PARA PROMOVER LA FORMACIÓN DE PROFESIONALES DE LA INDUSTRIA DEL SOFTWARE
}

Greiner, Cristina L(1).; Castro Chans, Beatriz(1,2); Dapozo, Gladys N. (1)

\section{Resumen}

El trabajo describe y revisa la ejecución del proyecto "La Lógica UNNE niveles y permite resolver problemas: Articulación orientada a mejorar la disponibilidad de RRHH para la industria del software de la región NEA", realizado en el marco del programa "La Universidad en el medio" de la Secretaría General de Extensión Universitaria.

El proyecto consistió en favorecer la articulación entre el nivel medio y la universidad, mediante acciones que colaboraran con la elección de las carreras de nivel superior, incrementar el número de inscriptos en carreras vinculadas con las TIC y disminuir las dificultades de los alumnos ingresantes, favoreciendo su permanencia en el sistema educativo superior; y de esta manera contribuir al desarrollo de la industria del software en la región.

Palabras claves: articulación universidad-nivel medio, ingresante universitario, vocaciones en TIC.

\section{Introducción}

La formación de recursos humanos en el sector de Software y Servicios Informáticos (SSI) de la Argentina es un tema prioritario en la agenda de la Educación Superior en nuestro país ya que la demanda del mercado laboral en la actualidad no es cubierta por los graduados locales. Una de las causas de esto, es la disminución en la elección de carreras informáticas por parte de los estudiantes. Como señalan Benotti, Echeveste y Schapachnik (2012): "Hay una problemática real que es el desbalance entre oferta y demanda de profesionales de Tecnologias de la Información y Comunicación (TIC), la cual debe solucionarse con urgencia debido a su criticidad para el desarrollo del pais." ${ }^{i}$

Considerando que las TIC contribuyen significativamente al crecimiento del PBI y de la productividad en la mayoría de los países, se comprende la relevancia de formar recursos humanos en esta área, con capacidad de innovación y de aprovechar el conocimiento global para aplicarlo en desarrollos específicos. En este sentido, "los sistemas educativos, y en particular las universidades que son responsables primarios de la formación de profesionales, se constituyen en el componente esencial para ser competitivos. ${ }^{\text {"ii }}$

(1) Departamento de Informática. Facultad de Ciencias Exactas y Naturales y Agrimensura (UNNE), Av.Libertad 5450, 3400, Corrientes, Corrientes, Argentina \{cgreiner, gndapozo\}@exa.unne.edu.ar (2) Dpto. Comunicación - Facultad de Humanidades - (UNNE) Sargento Cabral 2011,3400, Corrientes, Argentina beatriz.castrochans@comunidad. unne.edu.ar 
Conscientes de la tendencia decreciente de egresados y de la demanda insatisfecha de recursos humanos en ésta área, universidad, empresa y Estado implementan diferentes acciones para paliar este déficit, mediante becas, programas de calidad universitaria, de apoyo a la enseñanza de Informática, entre otras. ${ }^{\text {iii }}$

La mejora en la enseñanza en ciencias naturales en la Escuela Secundaria y el fortalecimiento del ingreso a carreras científicas y técnicas es una política pública prioritaria del Ministerio de Educación. En este sentido, se realizaron diversas acciones, tales como el Proyecto de Apoyo para Carreras de Ciencias Exactas y Naturales, Ciencias Económicas e Informática (PACENI), cuyo objetivo era promover que las universidades nacionales fortalezcan las condiciones institucionales, curriculares y pedagógicas para el mejoramiento de la inserción, promoción y rendimiento académico de los estudiantes del primer año de las carreras de Ciencias Exactas y Naturales, Ciencias Económicas e Informática; un ciclo de diálogo entre científicos y alumnos de escuelas secundarias, en el marco de jornadas “Ciencia ¿Qué me contás?”, con el objetivo de incentivar vocaciones científicas en los alumnos, fomentar el pensamiento crítico, desmitificar la figura del científico; el proyecto "La Universidad y la Escuela Secundaria. Mejora de la Formación en Ciencias Exactas y Naturales", entre otras. ${ }^{\text {iv }}$

En esta línea también trabaja la Fundación Dr. Manuel Sadosky, cuyo objetivo es favorecer la articulación entre el sistema científico tecnológico y la estructura productiva en todo lo referido a las TIC, mediante la implementación de distintos programas y proyectos orientados a mejorar la competitividad y hacer llegar los beneficios de las TIC a toda la sociedad. Entre ellos destaca el programa Vocaciones en TIC, que aplica diversas estrategias para despertar el interés de los jóvenes en seguir carreras afines. ${ }^{v}$

Esta problemática no es exclusiva de nuestro país, sino que existe una preocupación generalizada a nivel mundial pues se advierte que el suministro de recursos humanos formados en ciencia, tecnología, ingeniería y matemáticas (STEM - Science, Technology, Engineering and Mathematics) será menor que el necesario o deseable. Según un informe del Consejo Presidencial de Asesores en Ciencia y Tecnología (PCAST), en EE.UU. se requiere, en la próxima década, la producción adicional de aproximadamente 1 millón de graduados universitarios en los campos de STEM, situación complicada si se considera que menos del $40 \%$ de los estudiantes que ingresan a la universidad con la intención de especializarse en un campo STEM lo logran $^{\text {vi }}$, y que de 2008 a 2010, en los campos de ciencias de la computación e ingeniería, los doctorados otorgados cayeron un $7 \%$ y 4\%, respectivamente ${ }^{\mathrm{vii}}$. Otros países comparten este interés por enfocarse en temas relacionados a ciencia y tecnología, como un medio para el crecimiento económico. Brasil y Sudáfrica muestran altas tasas de crecimiento de C \& T, pero a partir de bases bajas. Entre las naciones más desarrolladas, Rusia realiza esfuerzos en la misma dirección, mientras que Israel, Canadá y Suiza son ejemplos de madurez y alto rendimiento en ciencia y tecnología. viii

Así como existen numerosas expresiones de preocupación, se dan diversos programas $^{\mathrm{ix}}$ y gran cantidad de artículos que analizan posibles soluciones y realizan propuestas, tales como aumentar la conciencia 
entre los estudiantes de la importancia y el atractivo de las carreras de STEM (las ofertas laborales ofrecen mejores remuneraciones), mejorar la educación en STEM desde los primeros niveles de educación ("Some STEM for All"), concentrar los esfuerzos sólo en los interesados o en los destacados (“All STEM for Some”) ${ }^{x}$, fortalecer la formación de los docentes de STEM y promover el incremento de los mismos mediante beneficios adicionales, entre otras. ${ }^{\mathrm{xi}}$

En este contexto, se llevó a cabo el proyecto "La Lógica UNNE niveles y permite resolver problemas: Articulación orientada a mejorar la disponibilidad de RRHH para la industria del software de la región $N E A$ ", en el marco del programa "La Universidad en el medio".

\section{Descripción del proyecto}

El objetivo general del proyecto consistió en favorecer la articulación entre nivel medio y universidad, mediante acciones que colaboraran con la elección de las carreras de nivel superior, incrementar el número de inscriptos en carreras vinculadas con las TIC (Tecnología de la Información y la Comunicación) y disminuir las dificultades de los alumnos ingresantes, favoreciendo su permanencia en el sistema educativo superior; y de esta manera contribuir al desarrollo de la industria del software en la región.

Por otra parte, los objetivos particulares fueron:

- Acercar a los alumnos del nivel medio, en especial de las escuelas técnicas con orientación informática de la Capital de Corrientes, experiencias prácticas acerca de las actividades propias del quehacer del profesional Informático.
- Incorporar los conceptos fundamentales de la matemática y de la lógica que permiten apoyar la estructura de conocimientos propios de la disciplina Informática.

- Consolidar en los alumnos el pensamiento lógico y los procesos de abstracción que se requieren para la resolución de problemas utilizando computadoras.

- Introducir a los alumnos en los conceptos y metodologías para la programación utilizando una herramienta sencilla y accesible al nivel de los alumnos.

- Repasar los fundamentos y afianzar los procedimientos propios de la comprensión lectora y su aplicación a la solución de problemas.

- Generar material didáctico para que las actividades puedan ser replicadas por los docentes del área Computación del establecimiento educativo.

Dadas las características del proyecto, se requería que los beneficiarios tuvieran ciertos conocimientos sobre las TIC, por lo que se seleccionó una institución educativa de nivel medio con orientación en Informática (usualmente conocidas como escuelas técnicas).

Para el logro de los objetivos previstos en el proyecto se conformaron equipos de trabajo formados por docentes, egresados y alumnos de la universidad para cada módulo a desarrollar.

La estrategia de abordaje se organizó en tres módulos que apuntaran a tres ejes clave:

- Módulo 1: Taller de Introducción 
a la Lógica, cuyo propósito era favorecer el desarrollo consciente del proceso del pensar y alcanzar un mayor nivel de aplicación de las habilidades mentales. En este módulo se realizaron actividades para favorecer una adecuada formación básica y tecnológica inicial con aportes de contenidos y procedimientos propios de la Lógica Clásica.

- Módulo 2: Taller de Interpretación y comprensión de consignas, cuyo propósito era fortalecer la capacidad de interpretación y análisis de consignas específicas pasibles de ser resueltas a través de soluciones algorítmicas. En este módulo se destacó el valor de la lectura como una de las prácticas socioculturales que posibilitan la apropiación de saberes y como herramienta fundamental para la construcción de conocimientos.

- Módulo 3: Taller de Capacitación en resolución de problemas con seudocódigo: la resolución de un problema mediante computadora consiste en el proceso que, a partir de la descripción de un problema, expresado habitualmente en lenguaje natural y en términos propios del dominio del problema, permite desarrollar un algoritmo de resolución a dicho problema. En este módulo se trabajó con los alumnos en la interpretación de consignas, discernimiento de datos necesarios e irrelevantes para el diseño de la solución, y la confección de los algoritmos de solución a los ejercicios propuestos.

\section{Metodología}

Previo acuerdo de trabajo con las autoridades y docentes de la institución educativa, se organizó la distribución temporal de los módulos de modo tal de no interferir con las clases de los estudiantes. Se selecciona- ron dos cursos del último año (aquellos con orientación informática), con un total de 40 alumnos.

Como primera actividad, se realizaron cuestionarios a los alumnos a fin de obtener un diagnostico situacional y perfil de los alumnos en cuanto al uso de herramientas informáticas, conocimientos sobre la resolución de problemas, y expectativas de formación posterior al nivel medio.

Por su parte, el personal docente responsable de cada módulo diseñó las actividades de enseñanza para su uso en las reuniones presenciales del Taller e instancias de trabajo no presencial de los participantes. Se elaboraron diapositivas, guías de actividades y de lectura a fin de coadyuvar a los participantes en el logro de los objetivos de enseñanza propuestos. Las actividades fueron diseñadas con el propósito de que los estudiantes construyan aprendizajes significativos y que la construcción de dichos saberes conlleven la adquisición de determinadas capacidades y competencias.

En el módulo 1: con el propósito de desarrollar la lógica clásica o bivalente como herramienta para construir conocimientos, tomar decisiones y desarrollar formas más complejas de pensamiento, se solicitó al alumno la resolución de ejercicios de lógica diseñados a tal efecto, sentando las bases para la resolución de problemas con computadoras. Cabe señalar, que se puso énfasis en los aspectos semánticos de los contenidos a estudiar y no en los sintácticos, a fin de no abrumar a los participantes con un simbolismo riguroso y excesivo, que podría llegar a ser un obturador para el logro de los objetivos de enseñanza propuestos. Por tal motivo, se incluyeron algunas actividades de lógica recreativa, similares a las que 
suelen presentarse en las páginas de entretenimientos de diarios y revistas. En este módulo, se trabajó para el logro de las siguientes capacidades y competencias:

o Identificar proposiciones de otro tipo de oraciones del lenguaje coloquial.

o Construir tablas de verdad de tautologías, contradicciones o contingencias, utilizando planilla de cálculo.

o Identificar proposiciones equivalentes.

o Utilizar argumentaciones válidas en la validación o refutación de conclusiones.

En el módulo 2: con el propósito de destacar la relevancia de la lectura comprensiva como estrategia mediadora y la evolución del lenguaje como elementos esenciales para el pensamiento abstracto y la capacidad para aprender, se utilizaron técnicas participativas grupales a fin de que los estudiantes ejercitaran la comprensión lectora y su aplicación a la solución de problemas, fortaleciendo su capacidad de interpretación y análisis de consignas. Las actividades propuestas permitieron, además, abordar aspectos clave del trabajo en equipo (liderazgo, coordinación, comunicación) y de la planificación (objetivos, metas, recursos, tareas). En este módulo, se trabajó para el logro de las siguientes capacidades y competencias:

o Interpretar consignas a través de la lectura comprensiva.

o Planificar actividades, estableciendo prioridades.

o Trabajar en equipo.
En el módulo 3: con el propósito de fortalezcan su capacidad de interpretación y análisis de consignas específicas pasibles de ser resueltas a través de soluciones algorítmicas se brindaron técnicas de análisis de problemas, y la posterior representación de la solución algorítmica en seudocódigo, utilizando una herramienta específicas, de baja curva de aprendizaje, para iniciar a los alumnos en la representación formal de algoritmos de solución a problemas. A tal fin, se presentaron a los alumnos los enunciados de problemas que trataban de reproducir situaciones comunes en su vida diaria. En este módulo se trabajó para el logro de las siguientes capacidades:

o Interpretar consignas (realizar un análisis)

o Determinar datos necesarios e irrelevantes para la resolución de problemas

o Representar formalmente una solución algorítmica

Asimismo, a partir de una invitación realizada por una importante empresa de desarrollo de software de la región (en Resistencia, Chaco) se organizó una visita a ocasión en la que los alumnos tuvieron la oportunidad de interactuar con los profesionales del lugar, y la posibilidad de inscribirse como aspirantes a una capacitación intensiva y avanzada, ofrecida por la mencionada empresa. Cabe señalar que esta actividad resultó altamente motivadora para el grupo de estudiantes que participó de la misma.

Al finalizar las actividades previstas en el proyecto, se realizaron encuestas a los alumnos a fin de recabar opiniones acerca del aporte de las acciones realizadas. 


\section{Materiales y resultados}

Como se expuso más arriba, el equipo coordinador del proyecto diseñó una metodología acorde a los estudiantes de nivel medio, considerando la edad, su vinculación con la tecnología (todos contaban con las netbooks que se entregaron a través del Programa Conectar Igualdad) y buscando motivarlos para el aprendizaje colectivo.

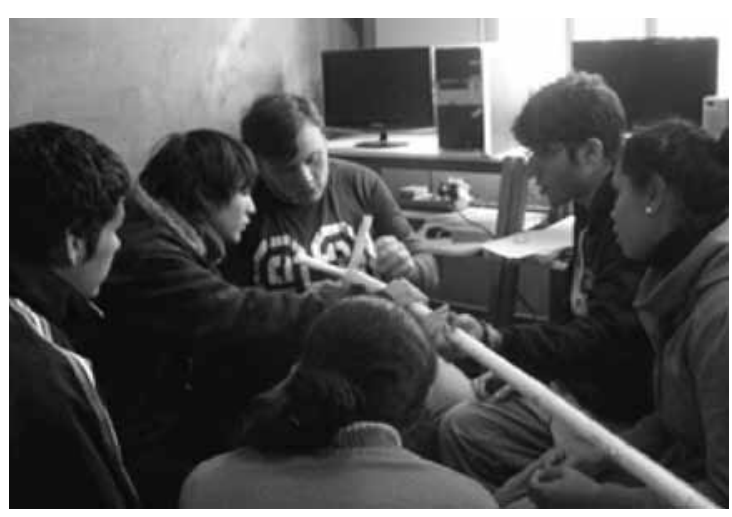

Figura 1: Comprensión y ejecución de consignas. Trabajo en equipo

Las actividades que incluían instancias lúdicas y de competencia, fueron las que lo- graron mayor adhesión y compromiso. En todos los casos, se incorporaron momentos de reflexión acerca de las lecciones aprendidas y sobre sus propias prácticas de aprendizaje.

Algunas de las actividades incluyeron la entrega de "recompensas" a los grupos, conforme el cumplimiento de las consignas. Más allá de que todos los grupos obtenían alguna, estas eran de carácter simbólico más que material (golosinas para la merienda) y se los motivaba a compartirla con sus pares, los alumnos se esforzaban por obtenerlo.

Al finalizar los módulos, se realizó una encuesta de opinión anónima a los estudiantes, a fin de conocer sus apreciaciones sobre las temáticas abordadas, la metodología de trabajo, cuáles son los temas sobre los cuales requieren mayor profundidad y cuáles los que consideran que van a necesitar para su desempeño laboral.

A continuación se presentan los resultados de dicha encuesta:

\section{Temas más novedosos}

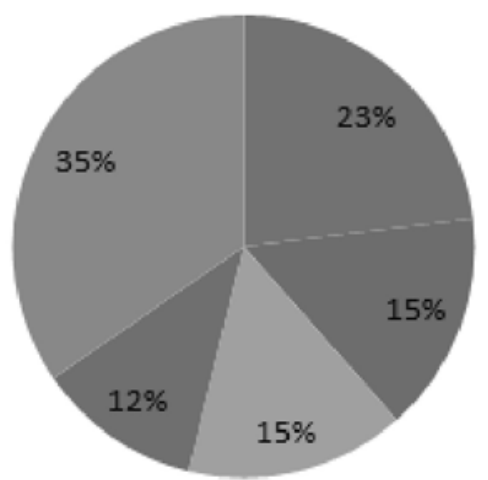

Figura 2: opinión sobre las temáticas abordadas

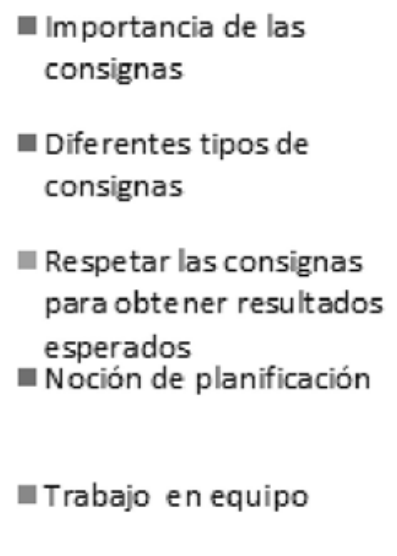




\section{Temas que necesitaría profundizar}

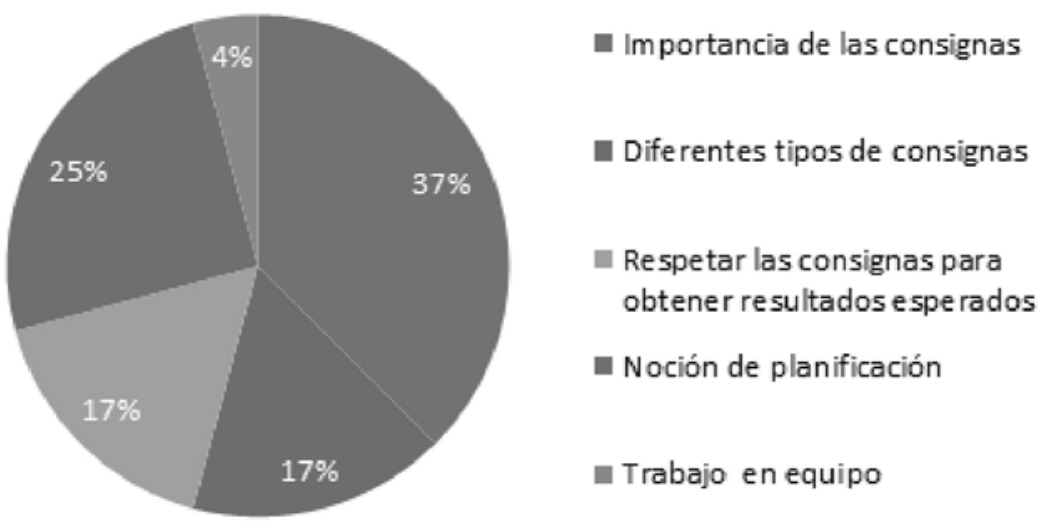

Figura 3: opinión sobre temas a profundizar

\section{Temas que se necesitan para trabajar}

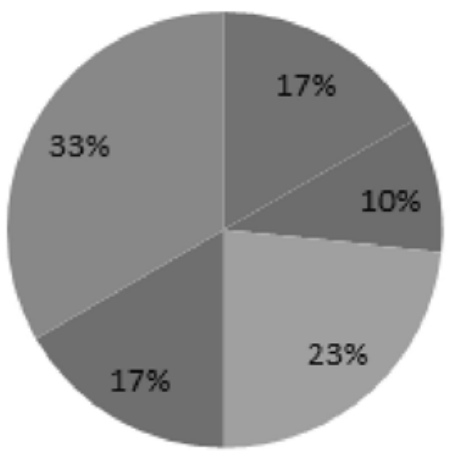

- Importancia de las consignas

n Diferentes tipos de consignas

- Respetar las consignas para obte resultados esperados

- Noción de planificación

- Trabajo en equipo

Figura 4: opinión habilidades requeridas en ambiente laboral

En los resultados de la encuesta se advierte que el trabajo en equipo alcanza el mayor porcentaje en relación a los temas que resultaron novedosos, como también, al consultar acerca de aquellos que serán necesarios al momento de insertarse en el mundo del trabajo (33\%). Sin embargo, no lo priorizan al momento de mencionar como un tema que requieren profundizar (4\%), con lo cual se podría suponer que desde la perspectiva de los estudiantes han alcanzado un manejo suficiente del mismo. El tema sobre el cual es necesario continuar profundizando es la importancia de las consignas (37\%), lo que daría lugar a pensar que a partir de la ejecución del proyecto los estudiantes han podido visibilizar la importancia de comprender las consignas para poder cumplir con ellas eficazmente. 
El trabajo en equipo es una condición necesaria para el desempeño laboral en el mundo actual, que implica comunicación dialógica, respeto a la diversidad de pensamiento, construcción de consensos, creatividad en la solución de problemas. La metodología con la que se trabajó se encuadró en esta modalidad lo cual ha sido claramente valorado por los estudiantes, quienes en un $62 \%$ consideraron que fue muy buena, y un $38 \%$ la señaló como buena.

\section{Conclusiones}

Desde la perspectiva del equipo docente, las actividades realizadas contribuyeron al fortalecimiento de las capacidades básicas de los alumnos beneficiarios en cuanto a la interpretación de consignas y resolución de problemas informáticos, el trabajo en equipo y la planificación y priorización de tareas. Asimismo, se motivó a los alumnos a continuar su formación profesional en esta línea, en el nivel superior de enseñanza.

Por otra parte, la interacción de los equipos docentes del nivel medio y superior contribuyó a potenciar las acciones en pro de fortalecer el desarrollo de la Informática en el nivel universitario.

Adicionalmente, el material didáctico generado, especialmente adaptado al perfil de los destinatarios del proyecto, podrá ser reutilizado posteriormente en actividades similares.
Se observó una mayor adhesión a las actividades lúdicas que a las de mayor formalidad, y, particularmente, se visualizó una alta motivación para realizar la visita a una empresa del medio, donde los alumnos pudieron observar el trabajo que se realiza, e intercambiar opiniones con profesionales del sector informático.

La intencionalidad de este proyecto, de contribuir a atenuar el pasaje del nivel medio a la universidad, a través de la provisión de herramientas conceptuales y metodológicas que contribuirán a su adaptación a las exigencias de la vida universitaria y a la permanencia y finalización exitosa de sus estudios.

Finalmente, la participación en el proyecto de docentes jóvenes, alumnos y recientes graduados, permitió el contacto de los mismos con una realidad en la que deben asumir una visión solidaria del conocimiento construido en la universidad. Estas experiencias van en la línea de formar profesionales críticos y conscientes de su compromiso social.

La interacción entre los niveles educativos para facilitar la articulación entre ambos es una tarea sobre la cual todos los actores deben comprometerse para resolver los graves problemas para el acceso y la permanencia en las carreras de nivel superior.

\section{Referencias}

i BENOTTI, Luciana; ECHEVESTE, María Emilia; SCHAPACHNIK, Fernando. (2012) "Despertando Vocaciones en Computación mediante el uso de autómatas de chat". Ponencia presentada en la 41 JAIIO Jornadas Argentinas de Informática, organizada por la Sociedad Argentina de Informática, realizada en La Plata (Buenos Aires, Argentina), del 27 al 31 de agosto de 2012. 
${ }^{i i}$ RED UNCI (Red de Universidades Nacionales con Carreras en Informática). (2008): "Formación de recursos humanos". Documento de trabajo. Disponible en: http:// redunci.info.unlp. edu.ar.

iii Marcelo Estayno; Gladys Dapozo; Liliana Cuenca Pletsch, Cristina Greiner. "Una experiencia de vinculación entre universidades y polos tecnológicos del NEA orientada a la promoción y consolidación de la industria del software en la región". 40 JAIIO, Jornadas Argentinas de Informática. UTN. Facultad Regional Córdoba. Córdoba. Argentina. Jornadas de Vinculación Universidad Industria. 40JAIIO - JUI 2011 - ISSN 1851-2518 - Página 26 a 35. 29 de Agosto al 2 de Septiembre de 2011.

iv Proyecto de Apoyo para Carreras de Ciencias Exactas y Naturales, Ciencias Económicas e Informática; http://portales.educacion.gov.ar/spu/calidad-universitaria/proyectos-de-apoyo/paceni/

Jornada “Ciencia ¿Qué me contás?”; http://portales.educacion.gov.ar/spu/la-universidad-y-laescuela-secundaria/ciencia-que-me-contas/

Proyecto "La Universidad y la Escuela Secundaria. Mejora de la Formación en Ciencias Exactas y Naturales"; http://portales.educacion.gov.ar/spu/noticias/se-abre-la-convocatoria-a-launiversidad-y-la-escuela-secundaria/

v Fundación Dr. Manuel Sadosky: Programas y Proyectos; http://www.fundacionsadosky.org.ar/ programas

${ }^{\mathrm{v}}$ Report to the president engage to excel: producing one million additional college graduates with degrees in science, technology, engineering, and mathematics. Washington: Executive Office of the President, President's Council of Advisors on Science and Technology (PCAST); 2012. Disponible en: http://www.whitehouse.gov/sites/default/files/microsites/ostp/pcast-engageto-excel-final_2-25-12.pdf

vii Robert Hummel, Patrick Cheetham. A DoD Perspective on STEM education. Synesis. Journal of Science, Technology, Ethics and Policy. Vol.3.25-36.

viii Science And Engineering Indicators - 2012; http://www.nsf.gov/statistics/seind12/pdf/ seind12.pdf

ix SHPE Foundation - Advancing Hispanic Excellence in Technology, Engineering, Mathematics and Science. http://www.shpefoundation.org/

${ }^{x}$ Refueling the U.S. innovation economy: Fresh Approaches to Science, Technology, Engineering and Mathematics (STEM) Education - por Robert D. Atkinson y Merrilea Mayo http:// www.itif.org/files/2010-refueling-innovation-economy.pdf )

xi Rising Above The Gathering Storm, Recuperado de: http://www.uic.edu/home/Chancellor/ risingabove.pdf) 